\title{
Effects of a concurrent chemoradiotherapy with S-1 for locally advanced oral cancer
}

\author{
SATOSHI HINO ${ }^{1}$, HIROYUKI HAMAKAWA ${ }^{1}$, YOUJI MIYAMOTO ${ }^{2}$, KAZUO RYOKE $^{3}$, JYOUJI SEKINE ${ }^{4}$, \\ AKIRA SASAKI $^{5}$, TETSUYA YAMAMOTO ${ }^{6}$ and THE ORAL CANCER STUDY GROUP OF CHUGOKU-SHIKOKU
}

\author{
${ }^{1}$ Department of Oral and Maxillofacial Surgery, Ehime University Graduate School of Medicine, Ehime 791-0295; \\ ${ }^{2}$ Department of Oral Surgery, Institute of Health Biosciences, The University of Tokushima Graduate School, \\ Tokushima 770-8504; ${ }^{3}$ Division of Oral and Maxillofacial Biopathological Surgery, Tottori University \\ Graduate School of Medical Sciences, Tottori 683-8503; ${ }^{4}$ Department of Oral and Maxillofacial Surgery, \\ Shimane University Faculty of Medicine, Shimane 693-8501; ${ }^{5}$ Department of Oral and Maxillofacial Surgery, \\ and Biopathology, Okayama University Dental School, Okayama 700-8350; ${ }^{6}$ Department of Oral \\ and Maxillofacial Surgery, Kochi Medical School, Kochi University, Kochi 783-0043, Japan
}

Received February 8, 2011; Accepted June 20, 2011

DOI: $10.3892 / \mathrm{ol} .2011 .347$

\begin{abstract}
A number of regimens composed of concurrent chemoradiotherapy (CCRT) have been attempted as radical or adjuvant therapies for locally advanced oral cancer. CCRT with S-1 is considered promising due to its efficacy and simplicity of application. Patients $(n=16)$ with locally advanced squamous cell carcinoma of the oral cavity were enrolled. Chemotherapy consisted of oral administration of S-1 $\left(65 \mathrm{mg} / \mathrm{m}^{2}\right)$ for 14 consecutive days followed by a 1 -week rest. Radiation treatment at a dose of 30 Gy in 15 fractions was administered concomitantly with $\mathrm{S}-1$. A course schedule of 3 weeks of treatment was applied twice. The overall response rate was $87.5 \%$. Median progression-free survival and median overall survival were 6.3 and 42.5 months, respectively. Although no grade 4 adverse events were observed, grade 3 adverse events, such as anemia (12.5\%), stomatitis (25\%) and anorexia (18.8\%) were present. Thus, CCRT with S-1 is an effective modality that can be safely conducted with minimal burden on patients.
\end{abstract}

\section{Introduction}

The therapeutic modality for oral cancer has not been standardized. For locally advanced cancers, multidisciplinary treatment, in which surgery, radiotherapy and chemotherapy are combined in various manners, is commonly applied. At present, surgery is considered to be the primary treatment modality, not only for an early stage cancer, but also for locally advanced

Correspondence to: Dr Satoshi Hino, Department of Oral and Maxillofacial Surgery, Ehime University Graduate School of Medicine, Shitsukawa, Toon, Ehime 791-0295, Japan

E-mail: sahino@m.ehime-u.ac.jp

Key words: oral cancer, S-1, concurrent chemoradiotherapy cancers. Among all cancer therapies, surgery is associated with the highest predictability, but its indication is affected by the patient's age, general medical condition, past histories or the extent of the lesion. At present, despite the availability of advanced reconstructive procedures are available, surgery remains associated with shortcomings including the likelihood of causing severe physical dysfunctions or adversely affecting the esthetic aspects of the body. The adverse effects associated with radiotherapy and chemotherapy, on the other hand, are often reversible and rarely leave functionally or esthetically untoward effects. However, these modalities should be applied for a certain length of time, during which the patient may suffer untoward effects. Moreover, predicting the therapeutic effects is difficult and the complete response (CR) rate is low when each modality is applied independently (1).

The oral cavity is an organ related to mastication, swallowing, respiration and facial appearance. Therapeutic approaches are required to achieve a favorable balance between minimal disturbances to its functions and esthetic appeal, and the most effective results. Previously, successive studies reported concurrent chemoradiotherapy (CCRT), in comparison to radiation monotherapy, in improving both the local control rates and prognosis (2-4). However, these studies were conducted as a radical therapy to avoid surgery, as a neoadjuvant therapy to reduce the tumor volume or as an adjuvant therapy following surgery. Findings of studies lack uniformity in their details, such as administration routes or anticancer regimens and methods, and dosages of radiation. CCRT with 5-fluorouracil (5-FU) or cisplatin (CDDP), anticancer agents also possessing the effect of enhancing sensitivity to radiation, outnumber other therapies (5-7). However, descriptions of the frequency and extent of their adverse effects and therapeutic results remain inadequate. It is generally known that pathological responses are positively correlated with clinical responses (8). Therefore, developing a regimen that is associated with high CR rates, can be applied safely and yet does not adversely affect the patient's quality of life (QOL) is crucial. 
S-1 (Taiho Pharmaceutical, Tokyo, Japan) is a 5-FU anticancer agent in which tegafur (a prodrug of 5-FU) is combined with gimeracil (an antagonist of 5-FU degrading enzyme DPD) and oteracil potassium (an agent that interferes with phosphorylation of 5-FU in the normal tissue of the digestive system). This composition is designed to increase the blood 5-FU concentration and AUC, potentiate its anti-tumor effect and reduce gastrointestinal toxicity. It can be administered via the oral route, which is a marked advantage $(9,10)$. The response rates to S-1 monotherapy applied to head and neck cancers were reported to be $46.2 \%$ (12/26 cases) or $28.8 \%$ $(17 / 59$ cases $)(11,12)$. In terms of the local control, the response rate increased $(48.1 \%, 25 / 52$ cases $)$. When applied to the oral cancers, the response rate was especially high (58.3\%, 7/12 cases). According to the Phase I clinical trial of CCRT with S-1 conducted by our research group (prior to the current study), the radiotherapy dosage was set at $60 \mathrm{~Gy}$ in 30 fractions and the recommended dosage of S-1 was $65 \mathrm{mg} / \mathrm{m}^{2} /$ day (13).

In the present study, a Phase II clinical trial was conducted to evaluate the efficacy and safety of CCRT with S-1 in treating locally advanced oral cancer patients.

\section{Patients and methods}

Eligibility. The participants in this study were 16 patients with histologically proven squamous cell carcinoma in the oral cavity. CCRT with S-1 was initiated during the period 2004-2009. The patients were also defined as those who had no metastatic lesion and no previous antitumor treatments. The specific eligibility criteria corresponded approximately to the prior study (14). The protocol was approved by the institutional ethics committee of each participating institution. At the start of treatment, the participating patients provided written consent.

Treatment. Chemotherapy consisted of oral administration of S-1 $\left(65 \mathrm{mg} / \mathrm{m}^{2} /\right.$ day $)$ for 14 consecutive days followed by a 1-week rest. Radiation treatment at a dose of $30 \mathrm{~Gy}$ in 15 fractions was administered concomitantly with S-1. A course schedule with a 3-week treatment was applied twice.

Toxicity and response assessment. Adverse events were evaluated according to version 2.0 of the National Cancer Institute-Common Toxicity Criteria (NCI-CTC). The antitumor effects were determined according to the Japan Society for Cancer Therapy Criteria as follows: CR for eliminating the tumor for $\geq 4$ weeks in all recognizable tumor lesions; partial response (PR) for $\geq 50 \%$ reductions in tumor size lasting for $\geq 4$ weeks; no change (NC) for findings ranging from tumor size reduction of $<50 \%$ to a tumor size increase of $<25 \%$ lasting for 4 weeks; and progressive disease (PD) for an increase in tumor size by $\geq 25 \%$ or the development of new lesions.

Statistical analysis. For the survival analysis, progression-free survival (PFS) and overall survival (OS) were estimated by the Kaplan-Meier method starting from the initiation of treatment. JMP ver7.0.1 (SAS Institute Inc.) was the software used for statistical analysis.
Table I. Patient characteristics $(n=16)$.

\begin{tabular}{lc}
\hline Characteristics & No. of patients \\
\hline Gender & \\
Male & 11 \\
Female & 5 \\
Mean age (years) & 73.5 \\
Stage & \\
II & \\
III & 2 \\
IVA & 7 \\
a & 7
\end{tabular}

Primary tumor site

Tongue 4

Lower gingiva $\quad 4$

Upper gingiva 3

Buccal mucosa $\quad 3$

Oral floor 1

Lower lip

Tumor differentiation

Well

8

Moderately

Poorly

5

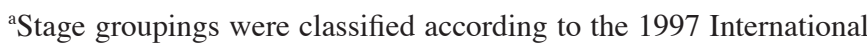
Union Against Cancer Criteria.

\section{Results}

Patients and treatment. A total of 16 patients, including 11 males and 5 females, were enrolled in this study. The patient characteristics are shown in Table I. The median duration of the observation was 7.4 months. The average total radiotherapy dosage was 58.6 Gy (range 30-70). Oral administration of S-1 for two courses was completed by $87.5 \%$ of the patients.

Adverse events. The adverse events observed during treatment are listed in Table II. Treatment was generally well tolerated throughout the study. Hematological toxicities were relatively mild. Severe conditions exceeding grade 3 were limited to leukocytopenia (6.3\%) and anemia (12.5\%). The most common adverse event present in patients was stomatitis (25\% at grades 3 or $4 ; 93.8 \%$ at all grades). Other severe adverse events evidenced at grade 3 or 4 were anorexia in $18.8 \%$ and diarrhea, rashes and fatigue in $6.3 \%$ each. Due to an increase in the severity of adverse events or refusal by the patient, $\mathrm{S}-1$ and/or radiation therapy was interrupted in 4 cases $(25 \%)$. No treatment-related death was observed during this study.

Clinical response. CR was achieved in 5 of 16 patients, PR in 9 patients and $\mathrm{NC}$ in 2 patients, while no incidence of $\mathrm{PD}$ occurred. The overall response and CR rates were 87.5 and $31.3 \%$, respectively. In terms of antitumor effects on the primary lesion, the CR rate increased in $37.5 \%$ of patients (Table III). The median duration was 6.3 months for PFS and 42.5 months for OS (Fig. 1). 
Table II. Adverse events during treatment.

\begin{tabular}{|c|c|c|c|c|c|c|}
\hline \multirow[b]{2}{*}{ Toxicity } & \multicolumn{6}{|c|}{ Grade } \\
\hline & 1 & 2 & 3 & 4 & $1-4(\%)$ & $3-4(\%)$ \\
\hline \multicolumn{7}{|l|}{ Hematological } \\
\hline Leukopenia & 5 & 5 & 1 & 0 & 68.8 & 6.3 \\
\hline Neutropenia & 2 & 3 & 0 & 0 & 31.3 & 0 \\
\hline Anemia & 9 & 3 & 2 & 0 & 87.5 & 12.5 \\
\hline Thrombocytopenia & 5 & 0 & 0 & 0 & 31.3 & 0 \\
\hline \multicolumn{7}{|l|}{ Non-hematological } \\
\hline T-Bilirubin & 4 & 0 & 0 & 0 & 25.0 & 0 \\
\hline AST & 0 & 1 & 0 & 0 & 6.3 & 0 \\
\hline ALT & 1 & 0 & 0 & 0 & 6.3 & 0 \\
\hline ALP & 1 & 0 & 0 & 0 & 6.3 & 0 \\
\hline Stomatitis & 1 & 10 & 4 & 0 & 93.8 & 25.0 \\
\hline Anorexia & 6 & 2 & 3 & 0 & 68.8 & 18.8 \\
\hline Nausea & 3 & 1 & 0 & 0 & 25.0 & 0 \\
\hline Vomiting & 0 & 1 & 0 & 0 & 6.3 & 0 \\
\hline Diarrhea & 0 & 1 & 1 & 0 & 12.5 & 6.3 \\
\hline Rash & 1 & 1 & 1 & 0 & 18.8 & 6.3 \\
\hline Pigmentation & 2 & 0 & 0 & 0 & 12.5 & 0 \\
\hline Fatigue & 3 & 4 & 1 & 0 & 50.0 & 6.3 \\
\hline
\end{tabular}

AST, aspartate aminotransferase; ALT, alanine aminotransferase; ALP, alkaline phosphatase. The adverse event was evaluated according to the NCI Common Toxicity Criteria, version 2.0.

Table III. Clinical efficacy of concurrent chemoradiotherapy.

\begin{tabular}{lcccccc}
\hline & CR & PR & NC & PD & Response rate (\%) & CR rate (\%) \\
\hline Local & 6 & 8 & 2 & 0 & 87.5 & 37.5 \\
Regional & 5 & 9 & 2 & 0 & 87.5 & 31.3 \\
\hline
\end{tabular}

$\mathrm{CR}$, complete response; $\mathrm{PR}$, partial response; $\mathrm{NC}$, no change; $\mathrm{PD}$, progressive disease.

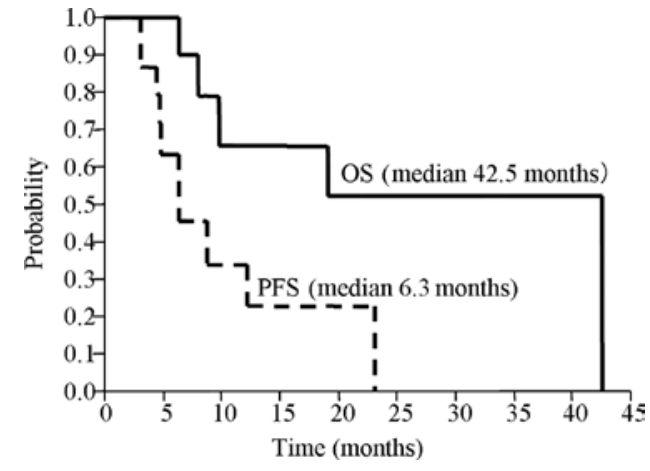

Figure 1. Kaplan-Meier curve for progression-free survival (PFS) and overall survival (OS). Median PFS and OS was 6.3 and 42.5 months, respectively.

\section{Discussion}

In this study, CCRT with S-1 was applied to 16 patients with locally advanced oral cancers to evaluate the efficacy and safety of the treatment. The participants mainly consisted of patients in Stage III or IV. Although 2 patients were classified as Stage II, their primary lesion corresponded to the so-called late T2 category. Surgery is the primary therapeutic option for patients in Stage I or II with so-called early T2. Therefore, our patient cohort was eligible for the purpose of this study.

Our group had previously conducted a clinical trial in which 41 patients with oral squamous cell carcinoma were treated with S-1 alone, and that the overall response and CR rates observed were 41.5 and $22 \%$, respectively (14). In this study, CCRT with S-1 resulted in overall response in $87.5 \%$ and CR in $31.3 \%$. In terms of the effect on primary foci, the CR rate was $37.5 \%$, indicating a high rate of local tumor control. Additive effects of S-1 on tumor cell killing by irradiation were also confirmed by in vitro study (15-17). Of the 11 patients in whom cancer was not cured, 2 refused additional treatment, 8 underwent salvage surgery, and selective arterial infusion therapy was possible in the remaining 
patient. These findings indicate that the current regimen can be applied safely without exacerbating the patient's general medical condition.

Neither therapeutic modality nor regimens of chemoradiotherapy for patients with oral cancer have been standardized. Platinum-based CCRT is most frequently chosen due to a favorable prognosis compared to other regimens with mitomycin C, 5-FU and BLM (4). Adelstein et al randomly administered radiotherapy alone, CCRT with CDDP, or CCRT with CDDP and 5-FU to 271 patients of squamous cell carcinoma of the head and neck region, and reported $\mathrm{CR}$ rates of 27.4, 40.2 and $49.4 \%$, respectively (2). Although CCRT with CDDP and 5-FU resulted in the highest CR, it is impossible to avoid surgery and preserve functions. Instead, problems of developing mucositis or leukopenia exceeding grade 3 persisted in approximately $40 \%$ of the patients. Due to its favorable local control rate, CCRT using superselective intra-arterial infusion has been widely applied to head and neck cancers. For locally advanced oral cancer, it has been reported that CCRT with CDDP and docetaxel using superselective intra-arterial infusion achieved a $90 \% \mathrm{CR}$ rate at the primary lesion (18). This modality has certain shortcomings, which include cumbersome catheter management in arterial infusion via the superficial temporal artery; a risk of cerebrovascular disorders or cranial nerve palsy when the Seldinger method is applied; a need for neck dissection when there is cervical lymphatic metastasis; and unreliability of the effects on distant metastasis that may exist latently or occur during treatment.

In the present study, the original intention was to accumulate the data from 55 patients in accordance with our statistical design; in reality, only 16 cases were enrolled. This may be explained not only by the diversity of therapeutic policies at individual institutions, but also by the differences in the circumstances under which therapy is conducted. There were discrepancies in the timing (initiation and completion) of S-1 administration and radiotherapy for some patients. Thus, it is likely that the synergistic effects of S-1 and radiotherapy may not have been completely reflected in these patients.

In spite of a high clinical efficacy, the CCRT of our regimen resulted in a relatively high incidence of stomatitis. When stomatitis becomes severe, the patient's QOL is compromised due to pain and readily provoked hemorrhage, and compliance to S-1 administration is threatened by difficulties in oral intake. Furthermore, the advantage of a minor rest level, which is possible only with oral chemotherapy, may be adversely affected. Studies $(19,20)$ are available that attest to the amelioration of CCRT-induced stomatitis through careful oral hygiene and care of the oral mucosa preceding the therapy. Such measures should be actively taken into consideration as part of therapeutic planning.

This study indicated that CCRT with S-1 is associated with a high clinical efficacy, suggesting that it can constitute a therapeutic option for locally advanced oral squamous cell carcinoma. In particular, it can be safely applied as pre-operative therapy with a minimum burden for the patient, while presenting the possibility of circumventing surgical procedures. The procedure is expected to constitute a base for regimens to achieve greater clinical efficacy in future.

\section{Acknowledgements}

The authors express their appreciation to Ehime, Tokushima, Tottori, Shimane, Okayama and Kochi Universities for their cooperation in enrolling the patients of the present study. The authors also extend their deep appreciation to the members of the Oral Cancer Study Group of Chugoku-Shikoku for their support.

\section{References}

1. Day TA, Davis BK, Gillespie MB, et al: Oral cancer treatment. Curr Treat Options Oncol 4: 27-41, 2003.

2. Adelstein DJ, Li Y, Adams GL, Wagner H Jr, Kish JA, Ensley JF, Schuller DE and Forastiere AA: An intergroup phase III comparison of standard radiation therapy and two schedules of concurrent chemoradiotherapy in patients with unresectable squamous cell head and neck cancer. J Clin Oncol 21: 92-98, 2003.

3. Wendt TG, Grabenbauer GG, Rödel CM, et al: Simultaneous radiochemotherapy versus radiotherapy alone in advanced head and neck cancer: a randomized multicenter study. J Clin Oncol 16: 1318-1324, 1998.

4. Browman GP, Hodson DI, Mackenzie RJ, Bestic N, Zuraw L, Cancer Care Ontario Practice Guideline Initiative Head and Neck Cancer Disease Site Group: Choosing a concomitant chemotherapy and radiotherapy regimen for squamous cell head and neck cancer: a systematic review of the published literature with subgroup analysis. Head Neck 23: 579-589, 2001.

5. Byfield JE, Calabro-Jones P, Klisak I and Kulhanian F: Pharmacologic requirements for obtaining sensitization of human tumor cells in vitro to combined 5-Fluorouracil or ftorafur and X rays. Int J Radiat Oncol Biol Phys 8: 1923-1933, 1982.

6. Richmond RC and Powers EL: Radiation sensitization of bacterial spores by cis-dichlorodiammineplatinum (II). Radiat Res 68: 251-257, 1976.

7. Taylor SG IV, Murthy AK, Caldarelli DD, et al: Combined simultaneous cisplatin/fluorouracil chemotherapy and split course radiation in head and neck cancer. J Clin Oncol 7: 846-856, 1989.

8. Kirita T, Shimooka H, Yamanaka Y, Tatebayashi S, Yamamoto K, Nishimine M and Sugimura M: Prognostic value of response to preoperative chemoradiotherapy and residual tumor grades in tongue carcinoma. Oral Surg Oral Med Oral Pathol Oral Radiol Endod 91: 293-300,2001.

9. Shirasaka T, Nakano K, Takechi T, et al: Antitumor activity of $1 \mathrm{M}$ tegafur-0.4 M 5-chloro-2,4-dihydroxypyridine-1 M potassium oxonate (S-1) against human colon carcinoma orthotopically implanted into nude rats. Cancer Res 56: 2602-2606, 1996.

10. Fukushima M, Satake H, Uchida J, et al: Preclinical antitumor efficacy of S-1: A new oral formulation of 5-fluorouracil on human tumor xenografts. Int J Oncol 13: 693-698, 1998.

11. Inuyama Y, Kida A, Tsukuda M, Kohno N and Satake B: [Early phase II study of S-1 in patients with advanced head and neck cancer. S-1 Cooperative Study Group (Head and Neck Working Group)]. Gan To Kagaku Ryoho (in Japanese) 25: 1151-1158, 1998.

12. Inuyama Y, Kida A, Tsukuda M, Kohno N, Satake B and S-1 Cooperative Study Group (Head and Neck Cancer Working Group): Late phase II study of S-1 in patients with advanced head and neck cancer. Gan To Kagaku Ryoho (in Japanese) 28: 1381-1390, 2001.

13. Sato $\mathrm{M}$ and Harada K: Phase I study of concurrent radiotherapy with S-1 for oral squamous cell carcinoma. Gan To Kagaku Ryoho (in Japanese) 33 (Suppl 1): 179-183, 2006.

14. Harada K, Sato M, Ueyama Y, Nagayama M, Hamakawa H, Nagahata S, Yoshimura Y, Osaki T, Ryoke K and Oral Cancer Study Group of Chugoku-Shikoku: Multi-institutional phase II trial of S-1 in patients with oral squamous cell carcinoma. Anticancer Drugs 19: 85-90, 2008.

15. Harada K, Kawaguchi S, Supriatno, Onoue T, Yoshida H and Sato M: Combined effects of the oral fluoropyrimidine anticancer agent, S-1 and radiation on human oral cancer cells. Oral Oncol 40: 713-719, 2004. 
16. Harada K, Kawaguchi S, Supriatno, Onoue T, Yoshida H and Sato M: Enhancement of apoptosis in salivary gland cancer cells by the combination of oral fluoropyrimidine anticancer agent (S-1) and radiation. Int J Oncol 25: 905-911, 2004.

17. Harada K, Kawaguchi S, Supriatno K, Kawashima Y, Yoshida H and Sato M: S-1, an oral fluoropyrimidine anti-cancer agent, enhanced radiosensitivity in a human oral cancer cell line in vivo and in vitro: involvement possibility of inhibition of survival signal, Akt/PKB. Cancer Lett 226: 161-168, 2005.

18. Mitsudo K, Tohnai I, Fuwa N, Furutani K, Nishiguchi H, Fukui T, Yamamoto N, Sugimura T, Saito M and Ueda M: Daily concurrent chemoradiotherapy with docetaxel (DOC) and cisplatin (CDDP) using superselective intra-arterial infusion via the superficial temporal artery for stage III and IV oral cancer: Possibility of organ preservation in advanced oral cancer. Toukeibu Gan (in Japanese) 32: 93-97, 2006.
19. Scully C, Epstein J and Sonis S: Oral mucositis: a challenging complication of radiotherapy, chemotherapy, and radiochemotherapy. Part 2: diagnosis and management of mucositis. Head Neck 26: 77-84, 2004.

20. Keefe DM, Schubert MM, Elting LS, et al: Updated clinical practice guidelines for the prevention and treatment of mucositis. Cancer 109: 820-831, 2007. 